\title{
Modelización de territorios ganaderos en la alta montaña al final del Neolítico: una integración de análisis espacial e información etnográfica*
}

\author{
Modeling livestock-herding territories in the high mountains at the end of the Neolithic: \\ an integration of spatial analysis and ethnographic information
}

\author{
Ermengol Gassiot Ballbè ${ }^{\text {, David Garcia Casas }}$, Joan Nunes Alonso ${ }^{c}$ y Guillem \\ Salvador Baiges ${ }^{a}$
}

\section{RESUMEN}

En los últimos años la investigación arqueológica en áreas de montaña de la península ibérica está empezando a llenar el vacío de información con extensas secuencias de ocupación durante el Holoceno. En el Pirineo se localiza una cantidad considerable de yacimientos del final del Neolítico/ Calcolítico con cronologías entre el 3350 y 2350 cal ANE. Este fenómeno parece vincularse con la consolidación de la explotación ganadera de las zonas alpinas y subalpinas. En este trabajo se analiza el patrón de dispersión de los yacimientos de esta época en el Parque Nacional de Aigüestortes i Estany de Sant Maurici, en el Pirineo central. Se definen mediante SIG áreas de influencia alrededor de los asentamientos y caminos óptimos para evaluar dos variables clave en cualquier práctica ganadera: la accesibilidad a los asentamientos y a los pastos. Con fines comparativos se analiza también el patrón de los yacimientos de época moderna y contemporánea. El estudio permite concluir diferencias significativas en la dispersión de los yacimientos de cada época con relación a los pastos y a los caminos.
2350 cal BC (Late Neolithic / Chalcolithic). This phenomenon seems to link with the consolidation of the livestock exploitation in the alpine and subalpine zones. This work undertakes a the dispersion pattern analysis of archaeological sites of this period in the National Park of Aigüestortes $i$ Estany de Sant Maurici, in the Central Pyrenees. Using GIS it defines influence areas around the sites and the optimal paths between them so as to evaluate two important variables in any livestock practice: the accessibility to the settlements and grazing areas. For comparative purposes the dispersion pattern of modern and contemporary sites is analysed as well. This study allows to conclude significant differences in the distribution of the sites both ancient and recent in relation to pastures and roads.

Palabras clave: Península Ibérica; Pirineos; Neolítico Final / Calcolítico; Alta montaña; Ganadería; Arqueología del paisaje; SIG; Etnografía.

Key words: Iberian Peninsula; Pyrenees; Late Neolithic / Chalcolithic; High mountain; Livestock; Landscape archaeology; GIS; Ethnography.

\section{INTRODUCCIÓN}

Durante la última década el conocimiento de la ocupación de las zonas de alta montaña de la penínpation during de Holocene. In the Pyrenees stands out the location of a considerable number of sites date to 3350 and

* Este trabajo está financiado por el proyecto "Modelización de los espacios prehistóricos de montaña. Un SIG del patrimonio arqueológico y los territorios pastoriles" (HAR2015-66780-P, Ministerio de Economía y Competitividad - Fondos FEDER) y el Parque Nacional de Aigüestortes i Estany de Sant Maurici.

a Grup de Recerca Tecnologies Digitals per a una Arqueologia Social 2017SGR243. Dpt. de Prehistòria. Universitat Autònoma de Barcelona. Edifici B. 08193 Bellaterra. Barcelona, España. Correos e.: EGB ermengol.gassiot@uab.cat https://orcid.org/0000-0003-0457-4805;

GSB guillem.salvador@uab.cat https://orcid.org/0000-0003-1248-7816

b Instituto de Ciencias del Patrimonio (Incipit) - CSIC. Avda. de Vigo s/n. 15705 Santiago de Compostela. A Coruña. España. Correo e.: david.garcia-casas@incipit.csic.es https://orcid.org/0000-0003-3490-9450

c Dpt. de Geografia. Universitat Autònoma de Barcelona. Edifici B. 08193 Bellaterra. Barcelona. España. Correo e.: joan.nunes@uab.cat https://orcid.org/0000-0002-6506-3518

Recibido 8-V-2019; aceptado 9-VII-2019.

Copyright: (C) 2020 CSIC. Este es un artículo de acceso abierto distribuido bajo los términos de la licencia de uso y distribución "Creative Commons Reconocimiento 4.0 Internacional" (CC BY 4.0) 
sula ibérica durante la Prehistoria ha evolucionado de forma considerable. Diversos proyectos de investigación, generalmente bajo un enfoque diacrónico y multidisciplinar, han incrementado de modo notable los registros arqueológicos del Sistema Central, la Cordillera Cantábrica y los Pirineos (Calastrenc et al. 2006; Díaz et al. 2016; González 2016; Laborda et al. 2017; Le Couédic 2010, 2012; Orengo et al. 2014; Rendu 2003; Rendu et al. 2016). Los avances son especialmente llamativos en los Pirineos, donde estudios recientes han puesto de relieve la existencia de yacimientos de diferentes períodos de la Prehistoria, principalmente del Neolítico.

Los datos actuales muestran como diferentes sectores del Pirineo axial albergaron la presencia de comunidades humanas familiarizadas con la ganadería, la agricultura y la alfarería desde como mínimo la segunda mitad del VI milenio cal ANE. Yacimientos de la provincia de Huesca, como Coro Trasito en el valle de Escuaín y Els Trocs en el de Benasque, situados a unos 1500-1600 m de altitud, son una buena prueba de ello (Clemente-Conte et al. 2016; Rojo et al. 2013). Con cronologías ligeramente posteriores al 5000 cal ANE para sus ocupaciones "neolíticas", la Cova del Sardo de Boí (Lleida) muestra un asentamiento humano en el fondo del valle de Sant Nicolau a $1780 \mathrm{~m}$ de altitud, en una de las zonas más abruptas del Pirineo (Gassiot et al. 2015; Gassiot 2016).

Sin embargo, la presencia humana en altitudes superiores durante el Neolítico no se estabiliza hasta la segunda mitad del período $\mathrm{y}$, especialmente, a partir del 3300 cal ANE. Con alguna excepción muy puntual, hasta después de esta fecha no se consolida la ocupación en áreas situadas alrededor o incluso por encima de la actual timberline. En las cuatro zonas de la cordillera que han sido objeto de intensas campañas de prospección de superficie durante la última década, después del Mesolítico la presencia humana no vuelve a ser visible arqueológicamente hasta la segunda mitad del IV milenio cal ANE e inicios del III milenio cal ANE (Dumontier et al. 2016; Gassiot 2016; Gassiot et al. 2017; Laborda et al. 2017; Le Couédic 2012; Orengo et al. 2014; Rendu 2003). En ese momento en las diferentes áreas estudiadas se disparan los indicios de ocupaciones humanas tanto en pequeñas cavidades y abrigos como al aire libre. A pesar de un notable descenso del número de yacimientos documentados en algunas zonas durante el II y I milenios cal ANE, la ocupación humana de espacios por encima los $2200 \mathrm{~m}$ de altitud continuará hasta los cambios en las prácticas ganaderas y los patrones de asentamiento del siglo XX.

El presente trabajo plantea el análisis espacial de estas ocupaciones a partir de los datos procedentes del Parque Nacional de Aigüestortes i Estany de Sant Maurici (en adelante PNAESM), el área de montaña de la península ibérica por encima de los $1800 \mathrm{~m}$ de altitud donde los registros de yacimientos del final del Neolítico/Calcolítico (entre 3400 y 2300 cal ANE) son más numerosos. A grandes rasgos, en esta época se constata la presencia de asentamientos humanos en áreas actuales de pastos subalpinos y, sobre todo, alpinos, lo que podría evidenciar la expansión de una ganadería posiblemente estacional hacia las zonas más altas. Desde una óptica amplia, aparentemente el patrón de dispersión de estos asentamientos prehistóricos es similar al que miles de años después se reproducirá en época contemporánea. Sin embargo, un análisis más fino evidencia diferencias significativas que alertan contra la tentación de asimilar las prácticas prehistóricas a una imagen derivada de la etnografía de la ganadería trashumante de finales del siglo XIX e inicios del siglo $\mathrm{XX}$, común en esta área de montaña. $\mathrm{El}$ análisis de similitudes y divergencias en los emplazamientos de los yacimientos de los distintos períodos ha de facilitar una mejor comprensión de algunas de las variables que guiaron el asentamiento humano en este sector del Pirineo al final del Neolítico/Calcolítico.

El desarrollo de nuevas metodologías y técnicas en la Arqueología incide directamente en el estudio de las diferentes actividades productivas pretéritas. Este hecho es evidente en el análisis de la ganadería prehistórica que, en los últimos años, ha efectuado progresos considerables también en las áreas de montaña. A grandes rasgos estos avances provienen de dos enfoques distintos. Por una parte, el conocimiento de nuevos yacimientos arqueológicos, con metodologías de excavación en constante desarrollo, y la aplicación de nuevas técnicas de análisis a los materiales arqueológicos están facilitando un volumen de información muy elevado con un nivel de detalle insospechado hace pocos años.

Por otro lado, en las dos últimas décadas se han llevado a cabo también estudios de los patrones de dispersión de yacimientos en el espacio y su relación con la configuración del medio físico y sus cambios a lo largo del tiempo (Carrer 2013; Garcia Casas 2018; González Álvarez 2016; ${ }^{1}$ ). Bajo una considerable diversidad teórica y metodológica estas investigaciones han prestado atención tanto a los pastos, lo que podrían haber sido los espacios productivos de montaña, como a las infraestructuras empleadas para la gestión y estabulación de los rebaños, así como para el refugio de las personas. Este último aspecto se ha revelado como un fértil campo de investigación en la arqueología en

\footnotetext{
${ }^{1}$ H. A. Orengo. Arqueología de un paisaje cultural pirenaico de alta montaña. Dinámicas de ocupación del valle del Madriu-PerafitaClaror (Andorra). Tesis doctoral inédita 2010, Institut Català d'Arqueologia Clàssica - Universitat Rovira i Virgili. Tarragona.
} 
áreas de montaña, dado su bajo nivel de urbanización y de impacto por parte de sistemas agrícolas intensivos. Ambos fenómenos permiten una mayor preservación y visibilidad en superficie de este tipo de vestigios constructivos que, en muchos estudios, conforman la base para la construcción de las secuencias arqueológicas de ocupación.

\section{MATERIALES Y MÉTODOS}

\subsection{Evidencias arqueológicas de ganadería en el área del Parque Nacional de Aigüestortes i Estany de Sant Maurici}

El PNAESM cubre un área de prácticamente 40.000 ha en el Pirineo de la provincia de Lleida, concretamente en el extremo oriental del batolito granítico de la Maladeta. Las cimas puntualmente alcanzan los $3.000 \mathrm{~m}$ de altitud y los fondos de valle se sitúan, en sus partes más bajas, sobre los $1.500 \mathrm{~m}$ de altura. Condicionada por el sustrato geológico y la orogenia alpina, el relieve muestra las trazas de la acción glaciar con un paisaje salteado de pequeños lagos, circos y valles en "U", delimitados por cumbres generalmente bastante abruptas y pedregosas. La orientación de los valles introduce variaciones sensibles especialmente en la humedad y el volumen de precipitación, hecho que junto con la altitud incide en los distintos ecosistemas presentes.

Hasta el año 2004 en esta zona del Pirineo no se habían registrado yacimientos arqueológicos de ninguna cronología. Las campañas de prospección arqueológica posteriores han permitido inspeccionar la superficie de casi todo el parque y documentar unos 350 asentamientos y otros restos arqueológicos. En su mayoría consisten en vestigios arquitectónicos de cabañas y recintos de dimensiones mayores, interpretados como cercados (Garcia Casas 2018; Gassiot 2016; Gassiot et al. 2016). Concretamente, los sitios con indicios arquitectónicos, al menos, de una estructura de estabulación representan más del $53 \%$ del total. La cifra se eleva a casi el $65 \%$, si se les incorporan las cabañas y espacios de hábitat al aire libre que podrían haber albergado a pastores. También se han localizado abrigos y pequeñas cavidades con trazas de ocupaciones pretéritas, posibles túmulos funerarios y estaciones de arte rupestre. Se dispersan por toda su extensión, sin evidenciar diferencias de densidad entre las distintas cuencas. En cambio, se constata una sobrerrepresentación de yacimientos entre los 2000 y $2400 \mathrm{~m}$ de altitud, coincidiendo con la posición actual del límite superior del bosque, el inicio de las áreas de pastos de altura y la cota de la mayoría de los circos y lagos. Esta preferencia altitudinal es especial- mente marcada entre los 2200 y 2400 m, una franja que representa el $27,8 \%$ de la superficie del PNAESM y que concentra el $48,1 \%$ de los yacimientos documentados.

Considerados como un todo, los yacimientos muestran parámetros relativamente homogéneos en su distribución espacial (Garcia Casas 2018; Gassiot 2016). Uno es la proximidad a fuentes de agua: el $75 \%$ se localizan a menos de $160 \mathrm{~m}$ lineales de una fuente de agua, río o lago, y la mitad a menos de $75 \mathrm{~m}$. Otro es su asociación con áreas de pasto: un poco más del $62 \%$ de los yacimientos se emplazan en áreas de pasto actual, un uso del suelo que cubre apenas el $29,9 \%$ de la extensión del PNAESM. Prácticamente el $77 \%$ de los demás está a menos de $50 \mathrm{~m}$ de estos pastos. Esta relación hay que tomarla con una cierta cautela, puesto que las secuencias de la paleovegetación evidencian una clara deforestación a partir de la Prehistoria reciente. Sin embargo no deja de ser llamativa la asociación general de los vestigios arqueológicos documentados con las áreas de herbazales. Este fenómeno podría responder también a un sesgo derivado del muestreo. Las prospecciones de superficie han tratado de cubrir la totalidad del terreno, pero la visibilidad en zonas de pastos es notablemente superior que en algunas áreas forestales (Gassiot et al. 2016). De otra parte, el binomio agua y hierba reúne dos de los factores clave en la ganadería de altura contemporánea que, presumiblemente, también lo fueron en el pasado.

Los sondeos y excavaciones en algunos de los yacimientos documentados ha facilitado una extensa serie de 86 dataciones de $A M S$ (del inglés Accelerator Mass Spectrometry) que proporcionan una secuencia de ocupación sin interrupciones para los últimos 10.000 años (Gassiot 2016; Garcia Casas 2018). Con todo, hay variaciones en la cantidad de contextos y yacimientos fechados. Por ejemplo, se constata un claro incremento del número de asentamientos documentados al final del Neolítico, entre el 3400 y el 2400 cal ANE, para luego observar una marcada caída en la misma cifra hasta prácticamente el cambio de era (Gassiot et al. 2016, 2017). La excavación en extensión del Abric de l'Estany de la Coveta I, la Cova del Sardo de Boí y el Abric de les Obagues de Ratera han documentado con detalle la reiteración de ocupaciones que se llevaron a cabo en estas pequeñas cavidades del PNAESM. En los tres yacimientos existen fases del final del Neolítico que, a su vez, se inscriben en secuencias de ocupación mucho más amplias que cubren desde distintos momentos del Mesolítico hasta épocas históricas (Gassiot et al. 2015; Gassiot 2016; Gassiot y Clemente 2018). Esta recurrencia de la ocupación humana en determinados lugares, se aprecia también en algunos sitios al aire libre, al menos en un caso también con la cronología del final del Neolítico

Trab. Prehist., 77, N. ${ }^{\circ}$ 1, enero-junio 2020, pp. 48-66, ISSN: 0082-5638

https://doi.org/10.3989/tp.2020.12246 
que se reproduce en otras áreas altas del Pirineo (Dumontier et al. 2016; Gassiot et al. 2017; Laborda et al. 2017; Orengo et al. 2014; Rendu 2003).

\subsection{Las ocupaciones pastoriles al final del Neolítico}

Con anterioridad al final de la Antigüedad e inicios de la Edad Media, el período mejor representado en la secuencia arqueológica del PNAESM es el comprendido entre el 3350 y el 2350 cal ANE (Garcia Casas 2018; Gassiot 2016; Gassiot et al. 2014, 2016, 2017). Los sondeos efectuados durante las campañas de prospección y algunas excavaciones en extensión han facilitado 14 fechas de C14 para un total de 10 fases de ocupación en 9 yacimientos distintos (Fig. 1). Cuatro son pequeños abrigos rocosos vinculados al aprovechamiento de oquedades en la base de bloques erráticos de origen glaciar (Fig. 1). Tres de las ocupaciones se vinculan también al aprovechamiento de pequeñas cavidades poco habituales en una zona granítica. Son pequeñas cornisas y una cueva de reducidas dimensiones. Las dos últimas proceden de yacimientos identificados al aire libre. La Coma d'Espós se vincula a un recinto definido por un zócalo de piedra y el Tuc deth Lac Redon, un nivel de ocupación localizado por debajo de una cabaña tardoantigua, reproduce un patrón documentado también en otros lugares de la cordillera (Orengo et al. 2014; Rendu 2003). Finalmente, el sondeo realizado en el Abric de la Girada Gran de Monestero proporcionó material lítico tallado similar al recuperado en otros contextos de este período que, por falta de material apto, no pudo ser datado de forma absoluta. Su adscripción al Neolítico final/Calcolítico es, por lo tanto, muy probable pero no segura. La tabla 1 ilustra brevemente los yacimientos considerados.

En su mayoría estos yacimientos se localizan por encima de los $2200 \mathrm{~m}$ de altitud. Únicamente VB-014, VB-016 y VB-023 están en cotas inferiores, a 1780 m, $1870 \mathrm{~m}$ y $1980 \mathrm{~m}$ respectivamente, en el tramo central del valle de Sant Nicolau. El techo lo marcan, por contra, NA-084 a $2410 \mathrm{~m}$ de altitud y ESP-008, a $2455 \mathrm{~m}$.

ESP-008, VB-014 y recientemente ESP-018 han sido excavados en extensión (Gassiot 2016; Gassiot y Clemente 2018; Gassiot et al. 2014; Mazzucco et al. 2019). En el primero se documentó un área de combustión con gran cantidad de carbón asociada a un nivel prácticamente limpio de otros restos arqueológicos y se interpretó como una ocupación relativamente puntual. En cambio, en VB-014 y ESP-018 la excavación en extensión documentó el uso de la cornisa y del abrigo rocoso de forma intensa y reiterada durante varios siglos. En ambos casos el espacio se articuló en torno a hogares y se descartaron herramientas líticas amortizadas y algunos fragmentos de recipientes de cerámica de dimensiones medianas y reducidas. El estudio de la industria lítica (Mazzucco et al. 2019) certifica la presencia de actividades cinegéticas, de procesamiento de productos vegetales y animales en un conjunto lítico con una importante presencia de materias primas del exterior de la cordillera. En los tres yacimientos las ocupaciones neolíticas forman parte de secuencias arqueológicas más amplias, y se han interpretado como producto del uso de las cavidades como lugar de habitación por parte de grupos que habrían llevado a cabo actividades de caza y pastoreo en la zona.

\subsection{La modelización arqueológica de territorios ganaderos del final del Neolítico}

La Arqueología, al basarse en registros fósiles, necesita construir procedimientos para conducir los procesos de inferencia de las prácticas sociales que los originaron. Esta es una tarea dual. Por una parte, dota a nuestra disciplina de un sentido explicativo más allá de la simple y tediosa descripción empírica. Por la otra, entraña numerosos riesgos siendo el principal, quizás, trasladar una analogía directa con el presente que borre las variaciones en esas mismas prácticas sociales a lo largo del tiempo y en diversos contextos geográficos. Las investigaciones recientes en las áreas de alta montaña ya han advertido del error de trasladar la imagen etnográfica de la trashumancia de época contemporánea directamente al pasado (Garcia Casas 2013).

Con todo, la construcción de modelos analíticos ha de facilitar el acceso a las realidades sociales pretéritas que constituyen el objeto de conocimiento de nuestra disciplina. En el presente trabajo planteamos una aproximación a las actividades humanas en el área de estudio al final del Neolítico y que causaron el incremento ya descrito en el número de vestigios arqueológicos y los primeros indicios, a nivel macro, de alteración de la cubierta vegetal (Gassiot et al. 2014). Para ello, estudiamos el comportamiento de dos variables a través de un análisis espacial del emplazamiento de los yacimientos de este período. La primera es su relación con las áreas actuales de pasto y la segunda, su vinculación con posibles vías de circulación. La pertinencia de estudiar ambas reside en la hipótesis de que la ganadería fue una actividad central en la ocupación humana de esta área de montaña al final del IV e inicios del III milenio cal ANE.

La existencia de toda actividad ganadera requiere de la alimentación del rebaño. En consecuencia, se justifica la premisa de que todo asentamiento ganadero, entendido como un lugar donde pernocta el ganado 

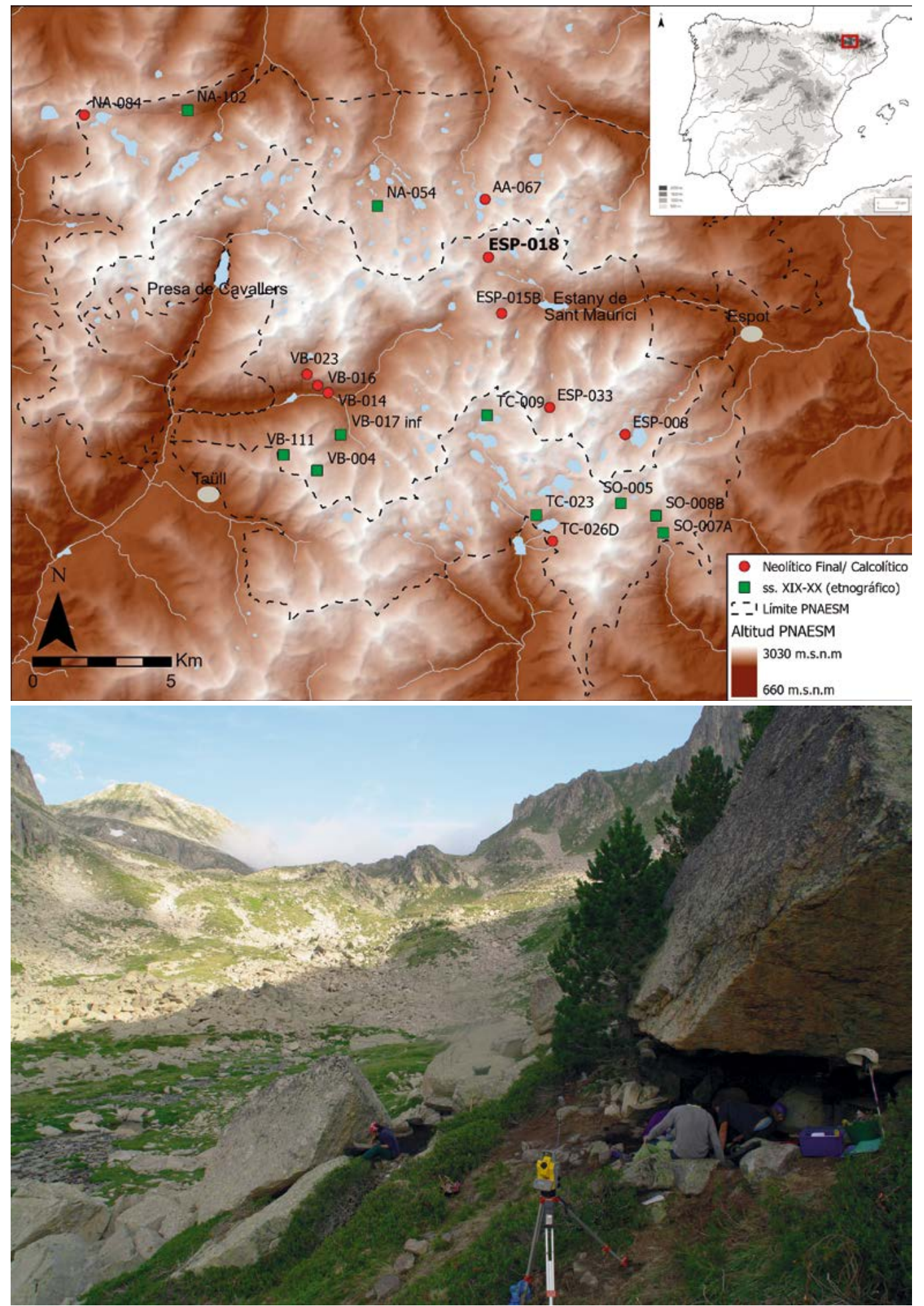

Fig. 1. Localización del Parque Nacional d'Aigüestortes i Estany de Sant Maurici (PNAESM) en la península ibérica y mapa del parque recortado sobre el MDE 5m/px del Institut Cartogràtic i Geològic de Catalunya. El Abric de les Obagues de Ratera, reproducido abajo durante su excavación el año 2015 se identifica como ESP-018 en el mapa (en color en la versión electrónica).

Trab. Prehist., 77, N. ${ }^{\circ}$ 1, enero-junio 2020, pp. 48-66, ISSN: 0082-5638

https://doi.org/10.3989/tp.2020.12246 
y quizás también las personas que lo cuidan, ha de mantener cierta cercanía con sus fuentes de aprovisionamiento de agua y comida. Algunos tipos de ganado pueden pastar en algunas áreas de bosque, pero es preferible buscar prados que son un nicho ecológico con gran cantidad de hierba disponible para el consumo animal (Landais y Balent 1995). En la montaña pirenaica el momento de floración en los meses de verano es el óptimo para su ingesta. Estos herbazales existían en el Pirineo de manera natural en el piso alpino, por encima de los 2400-2600 m aproximadamente, pero la apertura de bosques para aumentar las zonas de pasto disponibles los ha extendido al piso subalpino (1800-2200 m) antes poblado por árboles (Gómez 2008). Arqueológicamente también se documenta esta asociación entre la ganadería y el aumento de determinadas herbáceas que se vinculan a pastos (para ámbitos pirenaicos cercanos, véase p. ej. Galop et al. 2007; Pèlachs et al. 2017).

El acceso a los lugares es también una variable determinante para explicar una determinada actividad y su práctica. Si la actividad agrícola implica el acceso de la fuerza de trabajo al campo de cultivo, la actividad ganadera exige el desplazamiento del rebaño al lugar donde se alimenta y su mantenimiento allí durante cierto tiempo. Hay numerosas formas de evaluar el modo de acceso: la dificultad técnica, las restricciones sociales, el esfuerzo en términos de tiempo, trabajo o energía, etc. En ausencia de indicaciones sobre parámetros sociales de uso de un espacio, como p. ej. los regímenes de propiedad, el umbral de dificultad por encima del cual se excluye la realización de una determinada acti- vidad puede asimilarse a un tiempo determinado. A nivel espacial, esta variable se puede representar en términos de distancia. Cobra sentido definir zonas, $\mathrm{p}$. ej., alrededor de un asentamiento, donde es viable plantear prácticas como apacentar el ganado. La movilidad se define así desde un punto hacia su espacio circundante en todas las direcciones posibles o una parte considerable de ellas. Para escapar del sentido restrictivo del término "área de captación" (Vita-Finzi y Higgs 1970), entendemos este espacio como el entorno de influencia de un asentamiento. El coste de realizar las diferentes actividades configura el umbral de ese entorno que, en consecuencia, es variable.

Otra movilidad comporta un desplazamiento entre localidades equiparables por las acciones realizadas en cada uno de ellos. Casos paradigmáticos de esta movilidad son los desplazamientos nómadas que reubican los asentamientos y, por extensión, sus territorios circundantes de influencia. Aunque las diferentes situaciones históricas son variables, esta movilidad implica generalmente unos umbrales de distancia distintos a los de los desplazamientos cotidianos, así como, seguramente, la existencia de vías de circulación más acotadas. En el ámbito de lo que conocemos sobre la ganadería en zonas de alta montaña, se puede asociar tanto a los desplazamientos estacionales como a los movimientos de rebaños y pastores entre diferentes áreas de pasto dentro de una misma estación (Garcia Casas 2018; Le Couédic 2012; Rendu 2003; Violant 1949).

La utilización de aplicaciones de geoestadística y análisis espacial vinculada a la introducción de los Sistemas de Información Geográfica (SIG) en la Ar-

\begin{tabular}{|c|c|c|c|c|c|}
\hline Yacimiento & Nombre & Tipo & Intervención & Intervalo (cal ANE) & $N^{0}$ fechas \\
\hline AA-067 & Abric del Lac Major de Saboredo II & A & Sondeo & $2832-2462$ & 1 \\
\hline ESP-008 & Abric de l'Estany de la Coveta I & $\mathrm{A}$ & Excavación en extensión & $3309-3028$ & 1 \\
\hline ESP-015B & Abric del Portarró & A & Sondeo & $3007-2696$ & 1 \\
\hline ESP-018 & Abric de les Obagues de Ratera & A & Excavación en extensión & $3087-2505$ & $\begin{array}{l}4 \text { de una } \\
\text { fase }\end{array}$ \\
\hline ESP-033 & $\begin{array}{l}\text { Abric de la Girada Gran de } \\
\text { Monestero }\end{array}$ & $\mathrm{Al}$ & Sondeo & \multicolumn{2}{|c|}{$\begin{array}{c}\text { Cronología posible, inferida por } \\
\text { material lítico tallado }\end{array}$} \\
\hline NA-084 & Tuc deth Lac Redon & $\mathrm{Al}$ & Sondeo & $3260-2915$ & 1 \\
\hline TC-026D & Coma d'Espós & $\mathrm{Al}$ & Sondeo & $2886-2667$ & 1 \\
\hline VB-014 & Cova del Sardo & $\mathrm{Cc}$ & Excavación en extensión & $3484-2495$ & $\begin{array}{l}3 \text { para dos } \\
\text { fases }\end{array}$ \\
\hline VB-016 & Abric de les Covetes & $\mathrm{Cc}$ & Sondeo & $2571-2347$ & 1 \\
\hline VB-023 & Cova de Sarradé & $\mathrm{C}$ & Sondeo & $2566-2345$ & 1 \\
\hline
\end{tabular}

Tab. 1. Yacimientos del final del Neolítico/Calcolítico documentados en el Parque Nacional d'Aigüestortes i Estany de Sant Maurici. La cronología deriva de dataciones C14, calibradas mediante la curva INTCAL13 (Reimer et al. 2013) a un 95,4\% de probabilidad. Cuando hay varias fechas, se define el intervalo máximo del conjunto. Gassiot et al. $(2014,2016,2017)$ publican los detalles de las dataciones. A Abrigo; Al Aire libre; Cc Cueva - cornisa; C Cueva. 
queología se muestra como una herramienta fértil para avanzar en la modelización del acceso a los distintos lugares y las diferentes formas de movilidad en el pasado. Brevemente, detallamos los procedimientos empleados en este estudio.

\subsubsection{El cálculo de la extensión de los pastos dentro de las áreas de influencia}

Se han definido áreas circundantes a los asentamientos identificados en el PNAESM con cronologías del final del Neolítico/Calcolítico con el fin de cuantificar el pasto accesible para un determinado umbral de esfuerzo. El esfuerzo se ha asimilado al coste de desplazamiento traducido en tiempo, asumiendo que, por encima de ese umbral, disminuye el interés en aprovechar una cierta zona de pasto. Este umbral se ha situado en una hora de desplazamiento desde cada yacimiento a los pastos, asumiendo que allí pernoctaban las personas y, muy posiblemente, los rebaños.

El umbral se podría haber establecido tanto por encima como por debajo de la hora. La selección define un criterio convencional para cuantificar la extensión de una determinada superficie. La primera decisión metodológica ha sido escoger cómo modelizar espacialmente este umbral y la segunda cuantificar, dentro de él, la superficie de pastos. Detallamos seguidamente ambos procedimientos.

El desarrollo de los SIG permite cálculos de distancias de diferentes tipos. Sobre esta variable analítica desde la arqueología se han definido áreas de captación o de influencia alrededor de un asentamiento (p. ej., y entre muchos otros, Marín Arroyo 2008; Sánchez et al. 2016; White y Surface-Evans 2012). A grandes rasgos se han considerado dos concepciones del espacio. Una lo concibe como un elemento abstracto, sin contemplar cómo se conforma el espacio físico, ni variables como la elevación, el gradiente de las laderas y su orientación, etc. Operan a partir de la distancia geométrica o euclidiana. Vemos su aplicación en muchos mapas de densidad de puntos o en la definición de polígonos de Thiessen para definir patrones de dispersión de asentamientos. Fuera de zonas con relieves llanos y poco abruptos su aplicación es problemática. En cambio, los análisis basados en la segunda concepción espacial calculan la distancia real entre diferentes lugares. Para ello contemplan las características de la superficie sobre la que se produce el movimiento: su orografía, el tipo de terreno, la vegetación y otros factores naturales que pueden funcionar como barrera (como masas de agua), así como, en la medida de lo posible, variables de orden social (Conolly y Lake 2009; Wheatley y Gillings 2002).
Este trabajo opta por la segunda opción y calcula el coste de circulación por una superficie específica, asimilada a una superficie de coste, que ejerce una determinada fricción en función de unos criterios determinados. En el caso aquí tratado se considera que el condicionante principal de la movilidad en una zona de alta montaña procede del propio relieve. De esta forma, una superficie llana y horizontal facilita en mayor medida un desplazamiento que se vuelve más complicado y tedioso a medida que se incrementa su inclinación. Incluso se puede asumir que, por encima de un determinado gradiente, este desplazamiento se vuelve inviable. Obviamente, la cubierta vegetal puede afectar también de forma acusada la movilidad. Sin embargo, la vegetación puede ser el resultado directo de la utilización humana de un entorno y, por lo tanto, variar a lo largo del tiempo. Esta variable se ha obviado al no poder definirla con precisión en torno a cada asentamiento para el período de estudio. En cambio sí se ha considerado como factor limitante en los movimientos terrestres las masas de agua continentales o los lagos, muy abundantes en la zona.

En todo análisis de superficie de coste, ya sea para establecer áreas de influencia, caminos de mínimo coste, redes de caminos de mínimo coste o evaluar la accesibilidad, se suelen considerar relevantes los siguientes aspectos (véase, entre otros, Herzog 2013, 2014; Kantner 2012): a) la selección de los factores en la base del cálculo del coste de desplazamiento; b) la calidad y resolución de la información geográfica empleada para estimar los factores de coste; c) la función o modelo de coste escogida para generar la superficie de coste base del análisis; d) el algoritmo que calcula la superficie de coste acumulado, estrechamente ligado al software empleado, y que puede ser isotrópico o anisotrópico según considere o no la dirección de los desplazamientos; e) la mayor o menor capacidad de variar parámetros para refinar resultados tanto de la función de coste como del algoritmo de coste acumulado; y f) la validación de resultados mediante la comparación con evidencias arqueológicas conocidas.

También se señala (p. ej., Bevan 2011; Herzog 2013; Hudson 2012; Kantner 2012) la conveniencia de iniciar cualquier análisis de superficies de coste con un modelo simple, susceptible de refinarse más tarde con sucesivas variaciones o mejoras, y validable comparándolo con las evidencias empíricas disponibles. El presente ensayo prima la exigencia de calidad en los aspectos b) y f), y la simplicidad en a), c), d) y e). En otras palabras, se ha optado, en una primera prueba, por funciones de coste y algoritmos de coste acumulado simples que permitan obtener una primera aproximación a las áreas de influencia y posibles caminos "óptimos", en base al menor coste de desplazamiento,

Trab. Prehist., 77, N. ${ }^{\circ}$ 1, enero-junio 2020, pp. 48-66, ISSN: 0082-5638

https://doi.org/10.3989/tp.2020.12246 
entre los sitios objeto de análisis. Como señalan algunos autores (Hudson 2012), los caminos de mínimo coste se usan como aproximación a la accesibilidad, siendo secundario el coste real de recorrerlos.

Puede parecer que la elección de la función de coste es independiente, o cuando menos previa, a la elección del algoritmo de coste acumulado. Sin embargo, cuando el relieve, expresado mediante la pendiente, se toma como factor principal del coste de desplazamiento, ambas decisiones van unidas. Como la pendiente es, en sí misma, anisotrópica, la aplicación adecuada de la función de coste debería realizarse a través de un algoritmo de coste acumulado, que tenga en cuenta la dirección (cuesta arriba o abajo) del desplazamiento para calcular el coste. En su defecto, puede optarse, como en este caso, por una simplificación consistente en emplear un algoritmo de coste acumulado isotrópico a partir de una función de coste basada en la pendiente (como, p. ej. en Bevan 2011; Kantner 2012 y algunos casos de estudio analizados en Herzog 2013). La comparación posterior de los resultados obtenidos con ambos métodos suele mostrar diferencias secundarias más que discrepancias radicales.

La principal limitación del uso de una función de coste basada en la pendiente, mediante un algoritmo de coste isotrópico, es que solo considera el desplazamiento cuesta arriba. . Al estar ya calculado el coste correspondiente a una determinada pendiente todos los valores de la misma son positivos de la misma son positivos. Ello supone una cierta sobreestimación del coste empleado para determinar tanto las áreas de influencia en torno a los sitios como los caminos de mínimo coste entre sitios. Es decir, una hipótesis conservadora. Por el contrario, dicha simplificación solo valora, en parte, el carácter anisotrópico inherente a la pendiente, en su caso más desfavorable, que es el más relevante puesto que la reducción de coste que cabe suponer a la pendiente cuesta abajo solo tiene efecto en pendientes moderadas (inferiores a $12^{\circ}$, según la conocida fórmula de Langmuir 1995). Así, el efecto desfavorable al desplazamiento en las pendientes acusadas se ve reforzado por el uso exclusivo de pendientes positivas que hace el algoritmo isotrópico. Ello da lugar a costes acumulados desfavorables salvo en las pendientes moderadas, de manera que el camino de mínimo coste tenderá a coincidir con el obtenido mediante un algoritmo isotrópico aunque el valor del coste resulte sobreestimado.

Una vez establecidos como factores de coste el relieve y la exclusión de las masas de agua en tanto que barreras, la elección de la función de coste se ha basado en el criterio de obtener valores de tiempo de desplazamiento en función de la pendiente. Esa es la magnitud realmente significativa a la hora de definir áreas de influencia y de expresar la "distancia" entre sitios en áreas de montaña. Se han desechado pues opciones como el uso directo de la pendiente como valor de coste (p. ej., Hudson 2012), ya que produce valores de coste acumulado sin significado práctico, o como las funciones de coste que estiman el esfuerzo físico realizado por los sujetos que efectúan los desplazamientos. Dichas funciones dan lugar a estimaciones realistas del esfuerzo necesario y, por tanto, a superficies de coste ajustadas a la dificultad del desplazamiento. Sin embargo resultan poco operativas en términos territoriales para expresar áreas de influencia o distancias entre sitios.

Los análisis de superficies de coste en Arqueología han empleado diversas funciones de cálculo del coste en términos de tiempo de desplazamiento en función de la pendiente (véanse, para revisiones en detalle, Bevan 2011; Herzog 2014; Kantner 2012), ya sea mediante algoritmos de coste acumulado anisotrópicos o, en su versión simplificada (solo pendientes positivas), isotrópicos. Entre dichas funciones destacan las fórmulas de cálculo propuestas por Langmuir (1984), Tobler (1993) o Marín Arroyo (2008, 2009). La primera de ellas, implementada en la conocida función $r$-walk del programa de SIG GRASS, goza de un notable reconocimiento en este campo (Bevan 2011). Lo mismo ocurre con la de Tobler, una de las más empleadas en estudios de caminos mínimos (Herzog 2014 , p. ej., reporta su uso en 8 casos de estudio de un total de 15 analizados). Esas fórmulas tienen un componente anisotrópico que es sacrificado en la versión simplificada isotrópica, donde solo intervienen valores de pendiente positivos. Ello o bien resta sentido a su formulación inicial (especialmente la de la fórmula de Tobler, que utiliza una función exponencial) o bien la reduce a una función lineal de la pendiente positiva. En este caso la función solo emplea el tiempo base de desplazamiento en llano (constante de las fórmulas) y el coeficiente multiplicador de la pendiente. En este trabajo, se ha ensayado una función lineal sencilla, derivada empíricamente por uno de los autores (JNA, véase Anexo) a partir de datos de tiempo de recorrido y pendiente media de itinerarios excursionistas. Su formulación es la siguiente:

$$
\begin{aligned}
& \mathrm{t}=\mathrm{s} * 1,2532+9,1806 \\
& \text { donde } \\
& \mathrm{t}=\text { tiempo en minutos para recorrer } 1 \mathrm{~km} \\
& \mathrm{~s}=\text { pendiente en porcentaje }
\end{aligned}
$$

La fórmula ofrece valores intermedios (véase Anexo) respecto a la versión isotrópica de las funciones antes mencionadas, empleadas con el mismo fin. La fórmula de Tobler, que recoge mejor las variaciones locales del relieve y presenta valores más extremos donde la pendiente es muy acusada podría parecer más oportuna. La propuesta por Marín Arroyo $(2008,2009)$ 
proporciona resultados más suavizados, y produce en general tiempos inferiores, sobre todo en las pendientes más altas. Ello incrementa la distancia que se puede recorrer dentro de una determinada magnitud de tiempo. Los resultados obtenidos al aplicar las tres fórmulas en algunos yacimientos se han cotejado con el tiempo real necesario para recorrer determinadas distancias en el terreno actual. Ello ha permitido comprobar que los valores de la fórmula de Nunes (véase Anexo), intermedios a los de las otras dos, se ajustan mejor a los tiempos actuales. La fórmula de Langmuir produce valores de tiempo similares a los de la fórmula propuesta para valores moderados de pendiente (hasta $40 \%$ ). En pendientes más acusadas da tiempos sensiblemente inferiores, intermedios también entre los producidos por las fórmulas de Marín Arroyo y Tobler.

En el presente ensayo se ha empleado el software ArcGIS 9.5. En concreto, el algoritmo de coste isotrópico (función Cost Distance), que implementa el conocido algoritmo de Dijkstra. Este último produce el valor de coste acumulado para cada celda del ráster de la superficie de coste inicial, al tiempo que registra para cada una la de menor coste hacia la cual moverse. De ese modo es posible posteriormente obtener el camino de mínimo coste retrocediendo de cada celda destino a cada celda origen. El proceso podría haberse realizado con otros programas, ya que la implementación del algoritmo de coste acumulado isotrópico es parecida en todos. Los análisis de superficie de coste en Arqueología emplean también programas como IDRISI y GRASS (Gietl et al. 2008; Herzog 2014), así como softwares desarrollados por los propios autores. Sin embargo predomina el empleo de ArcGIS (p. ej. en 11 de los 15 casos estudiados en Herzog 2014).

El procedimiento seguido ha consistido en: i) calcular la pendiente en porcentaje a partir del Modelo Digital de Elevaciones (MDE, DEM en inglés) distribuido por el Institut Cartogràfic i Geològic de Catalunya, con un ancho de píxel de $5 \mathrm{~m}$, lo cual asegura un alto nivel de detalle; ii) generar a continuación, mediante la fórmula propuesta, una superficie de coste o fricción para toda el área de estudio; iii) añadir el efecto barrera de las masas de agua (principalmente lagos) asignándoles un valor de coste muy elevado con el fin de introducir un factor limitante a la circulación en esas áreas que aparecen totalmente llanas en el MDE; iv) obtener la superficie de coste acumulado a partir de cada uno de los sitios arqueológicos objeto de estudio mediante el algoritmo de coste isotrópico de ArcGIS.

Este procedimiento genera un mapa continuo del tiempo de desplazamiento en minutos desde el yacimiento (Fig. 2). Sobre esa base se definen líneas de isocoste de 60 minutos que engloban toda la superficie entorno al mismo hasta una hora de distancia. Este cálculo tiene dos imprecisiones: excluye la vegetación (véase más arriba) y la velocidad de desplazamiento. Esta depende de la composición y estado físico de las personas del grupo, de la carga transportada, en su caso, del tipo de ganado, la meteorología, etc. En cambio, la modelización establece áreas comparables en torno a los yacimientos, al margen de si los recorridos desde los mismos suponen 60,50 o 75 minutos.

Finalmente, para cada yacimiento, se ha calculado la superficie de pasto definida por la línea de isocoste de una hora, mediante el Mapa de Cobertes del Sòl de Catalunya (MCSC-4) realizado por el Centre de Recerca Ecològica i Aplicacions Forestals (CREAF 2013) (Fig. 3). Se han contemplado solo las áreas de pasto y de cultivos actuales al reflejar un paisaje vegetal cercano a los máximos de antropización de los últimos 10.000 años (Catalan et al. 2013). Las imprecisiones se han considerado asumibles, dado que se pretende comparar los potenciales pastos entorno a los yacimientos más que valores absolutos de la realidad pretérita. La superficie se ha calculado aislando una única capa de pasto y rasterizándola en píxeles de $5 \mathrm{~m}$ de ancho.

\subsubsection{La definición de posibles caminos}

Los caminos se han definido sobre la misma base: una superficie de coste caracterizada mediante el algoritmo ya detallado sin las áreas lacustres. Siguiendo el principio teórico de los caminos óptimos, se han definido como el recorrido de menor coste entre dos puntos, una vez definida una superficie de coste acumulado. Su trazado se ha efectuado igualmente mediante ArcGIS 10.5 (función Cost Path).

La decisión más relevante del análisis fue definir los orígenes y destinos de los caminos, dado que se pretendía observar la relación de los yacimientos con las hipotéticas vías de circulación y no la circulación entre ellos. En las inmediaciones del PNAESM apenas se conocen yacimientos prehistóricos que pudieran haber sido los nudos de la red. Este papel se ha asignado a los núcleos de población estable actuales situados alrededor del parque. En primer lugar, a menudo son asentamientos con una historia muy prolongada. Además se emplazan en los principales valles circundantes al PNAESM y conectan sectores por los que, sin duda, transcurrió un poblamiento prehistórico. Por último, los caminos resultantes pueden explicar la movilidad en épocas más recientes. Se han trazado las vías óptimas entre las poblaciones de Espot, València d’Àneu, Salardú, Arties, Vielha, Vilaller, Taull, Boí, Torre de Capdella, Llessuí, etc cuyo recorrido cruza partes del PNAESM. Como su definición sobrepasa con mucho la superficie del parque, se han simplificado los cálculos empleando el MDE de $15 \mathrm{~m}$ de ancho de píxel (Garcia Casas 2018). 


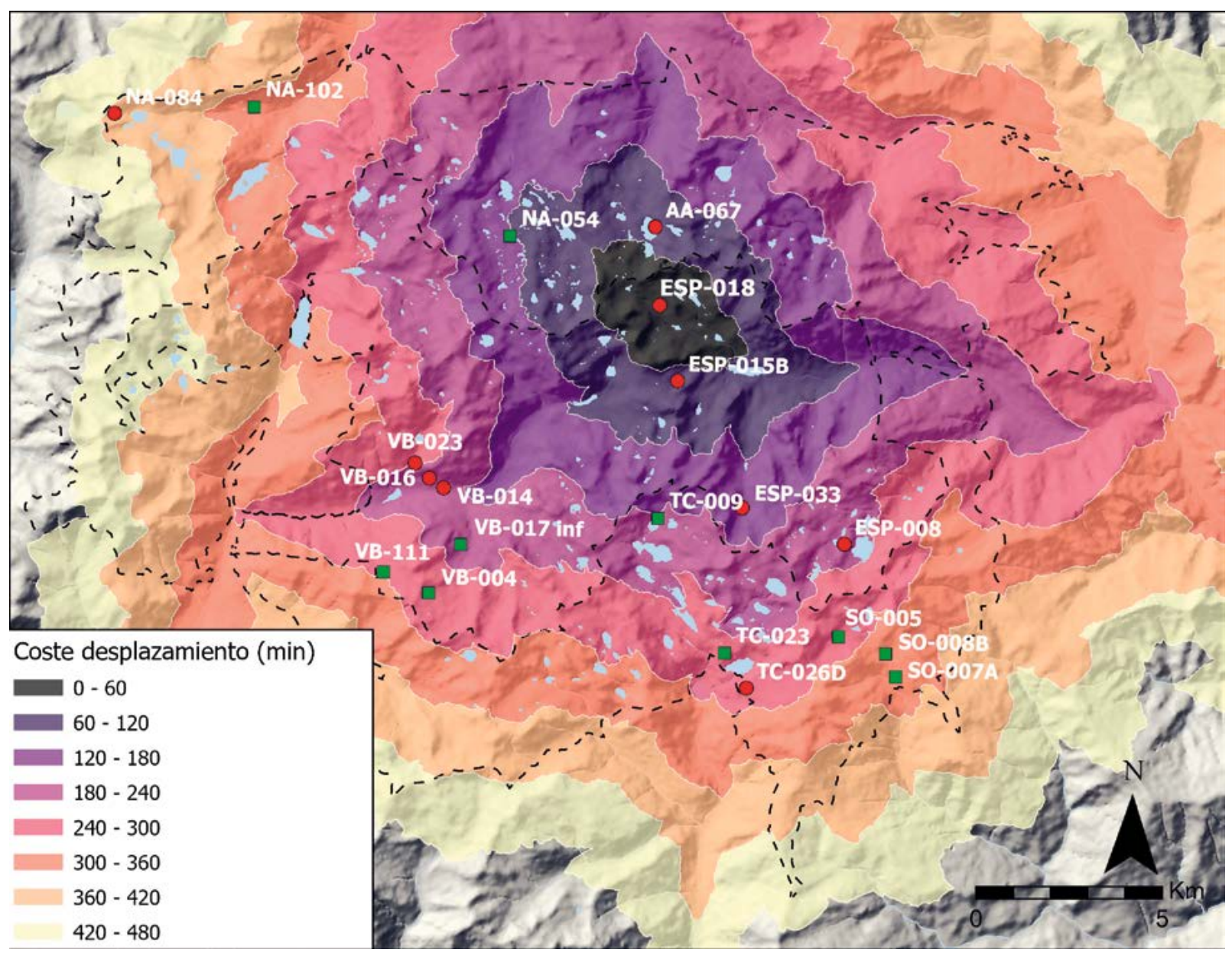

Fig. 2. Cálculo de las distancias de coste desde el Abric de les Obagues de Ratera (ESP-018). Las líneas de isocoste distinguen intervalos de 30 minutos. En línea discontinua, los límites del Parque Nacional d’Aigüestortes i Estany de Sant Maurici (en color en la versión electrónica).

El grado de coincidencia es muy elevado entre la red de caminos obtenida, los caminos y senderos actuales y las vías pecuarias que figuran en la cartografía de referencia de ámbito autonómico (Departament d'Agricultura 2013). Por otra parte, se han eliminado caminos que transcurrían por zonas de difícil acceso, resultado de operar con mapas más imprecisos y con píxeles mayores.

Una vez trazados los distintos caminos óptimos, se ha calculado la distancia de cada yacimiento a la ruta más cercana. Este valor se estableció a partir del tiempo y no de la distancia lineal o euclidiana. Para ello, y empleando de nuevo el MDE con un píxel de $5 \mathrm{~m}$, se han calculado las áreas de distancia de coste alrededor de los diferentes caminos en su trazado dentro del PNAESM. El mapa resultante ha calculado en minutos el recorrido entre el emplazamiento de cada yacimiento y el camino más cercano (Fig. 4).

\subsection{La comparación con yacimientos de época contemporánea}

En ocasiones en Arqueología se obtienen valores para variables difíciles de ponderar: el tiempo de trabajo que implica una determinada producción, el rendimiento de una acción o el grado de visibilidad de un emplazamiento hacia su territorio circundante. En nuestro caso sucede lo mismo. Como resultado de las acciones expuestas en los apartados precedentes contamos con superficies de pasto en áreas acotadas alrededor de los yacimientos prehistóricos y con las distancias entre estos y una red de caminos teóricos. No obstante, traducir los valores obtenidos a una comprensión de dinámicas sociales pretéritas sigue siendo una actividad compleja.

Para facilitar esta tarea, se han calculado los mismos valores para una cifra idéntica de asenta- 


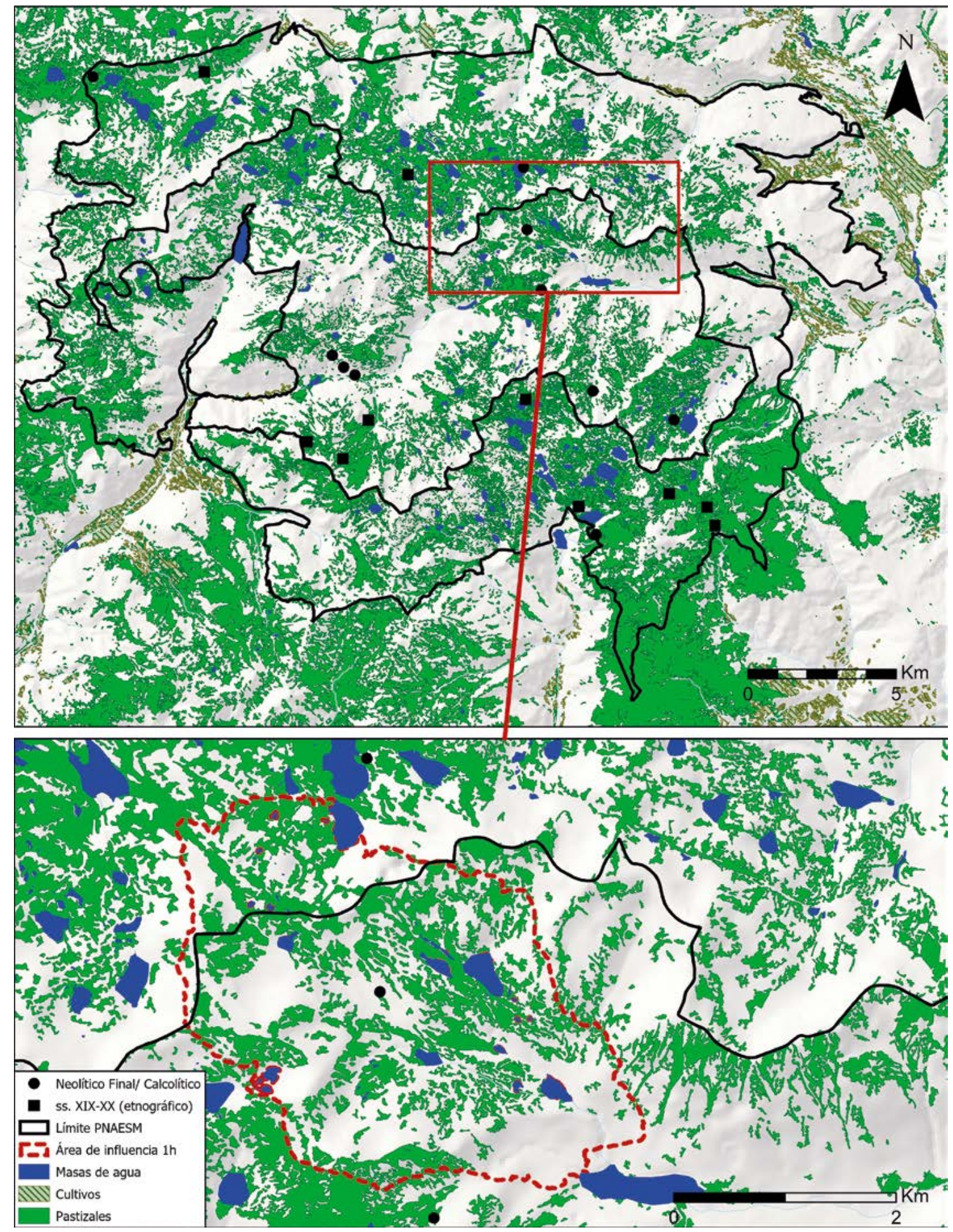

Fig. 3. Parque Nacional d'Aigüestortes i Estany de Sant Maurici (delimitado en negro; los lagos en sombreado continuo gris oscuro). Mapa de áreas de pasto (gris claro) y cultivos (rallado) generado a partir del Mapa de Cobertes del Sòl de Catalunya (MCSC-4) sobre el MDE de $5 \mathrm{~m} / \mathrm{px}$ del Institut Cartogràtic i Geològic de Catalunya. Abajo detalle de la extensión de pastos en el área de influencia a 1 hora del Abric de les Obagues de Ratera (en color en la versión electrónica, azul para los lagos, verde para las áreas de pasto). 


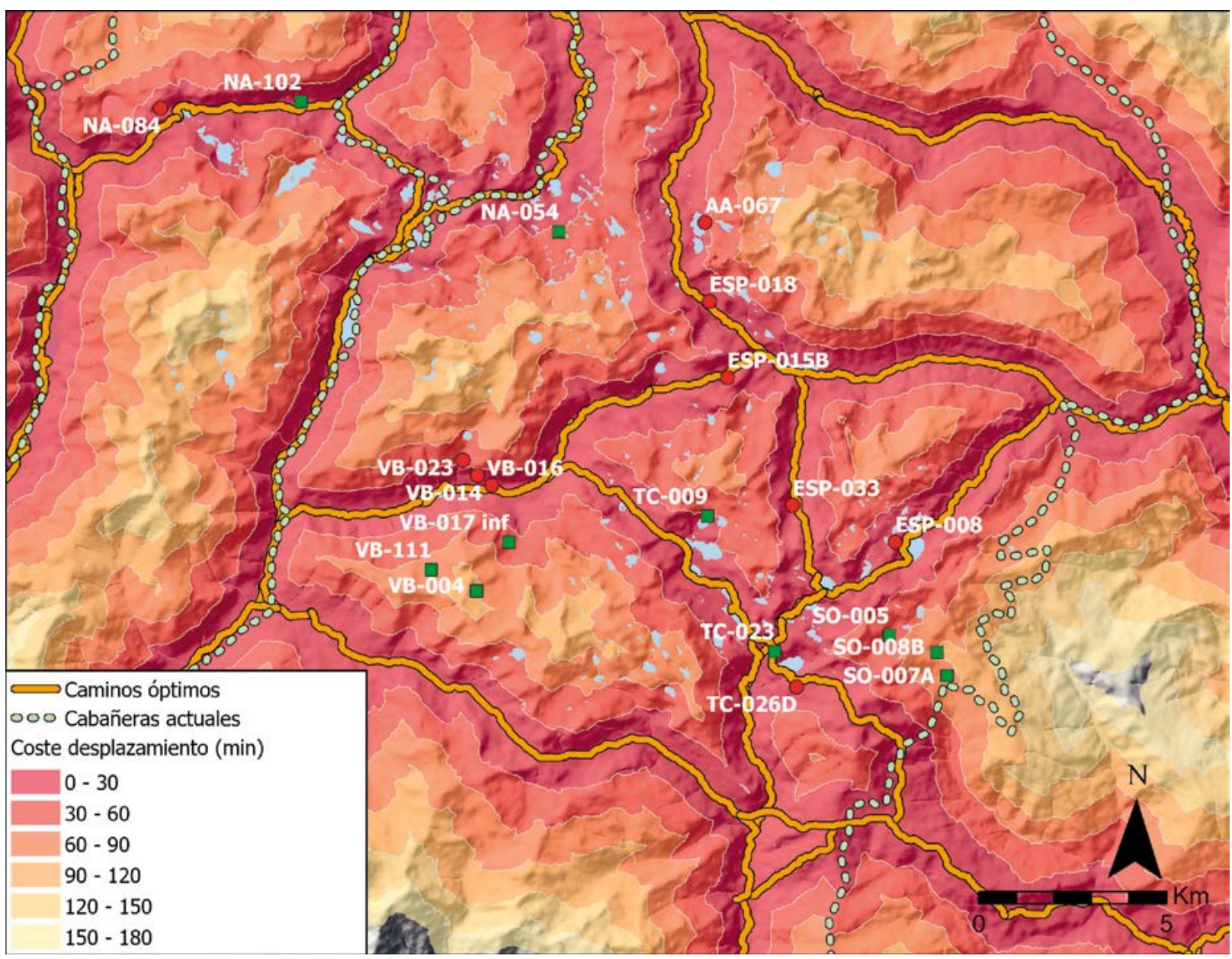

Fig. 4. Parque Nacional d'Aigüestortes i Estany de Sant Maurici (PNAESM). Mapa de caminos óptimos y las cabañeras actuales. Se señalan también las distancias de coste con respecto los caminos óptimos, hasta un máximo de 180 minutos (en color en la versión electrónica).

mientos con cronologías de los últimos 200 o 250 años. Se han seleccionado yacimientos con dataciones absolutas de este periodo, así como otros cuyos materiales superficiales (hojas de navajas de hierro) y su arquitectura permiten dicha asignación (Gassiot 2016; Garcia Casas 2018). Además se han priorizado aquellos claramente relacionables con las actividades pastoriles descritas por las fuentes etnográficas para esta zona del Pirineo (Violant 1949, 2001; Vilarrassa 1981). Según explican la gestión de los rebaños está orientada a la explotación al máximo de los pastos, representando los asentamientos de altura uno de los extremos de una ganadería estacional. La comparación pretendía poder detectar si existían diferencias entre ambos conjuntos en su relación con las vías de paso y en el acceso a las zonas de pasto.

\section{RESULTADOS}

\subsection{Asentamientos y áreas de pasto}

Siguiendo los procedimientos descritos, se ha calculado la superficie de pasto (y cultivos) en un desplazamiento radial de una hora en torno a cada lugar considerado. Una vez conformada una superficie de coste, se han delimitado las distancias de una hora alrededor de los 10 asentamientos con cronologías del final del Neolítico y Calcolítico (Fig. 5). Estas distancias han considerado la incidencia de la orografía en la facilidad y velocidad de los desplazamientos a pie de una o varias personas y dejando de lado la incidencia de la vegetación. Las delimitaciones obtenidas son orientativas pero constantes entre los diferentes yacimientos, por lo que sirven de base comparativa entre ellos (Tab. 2). 


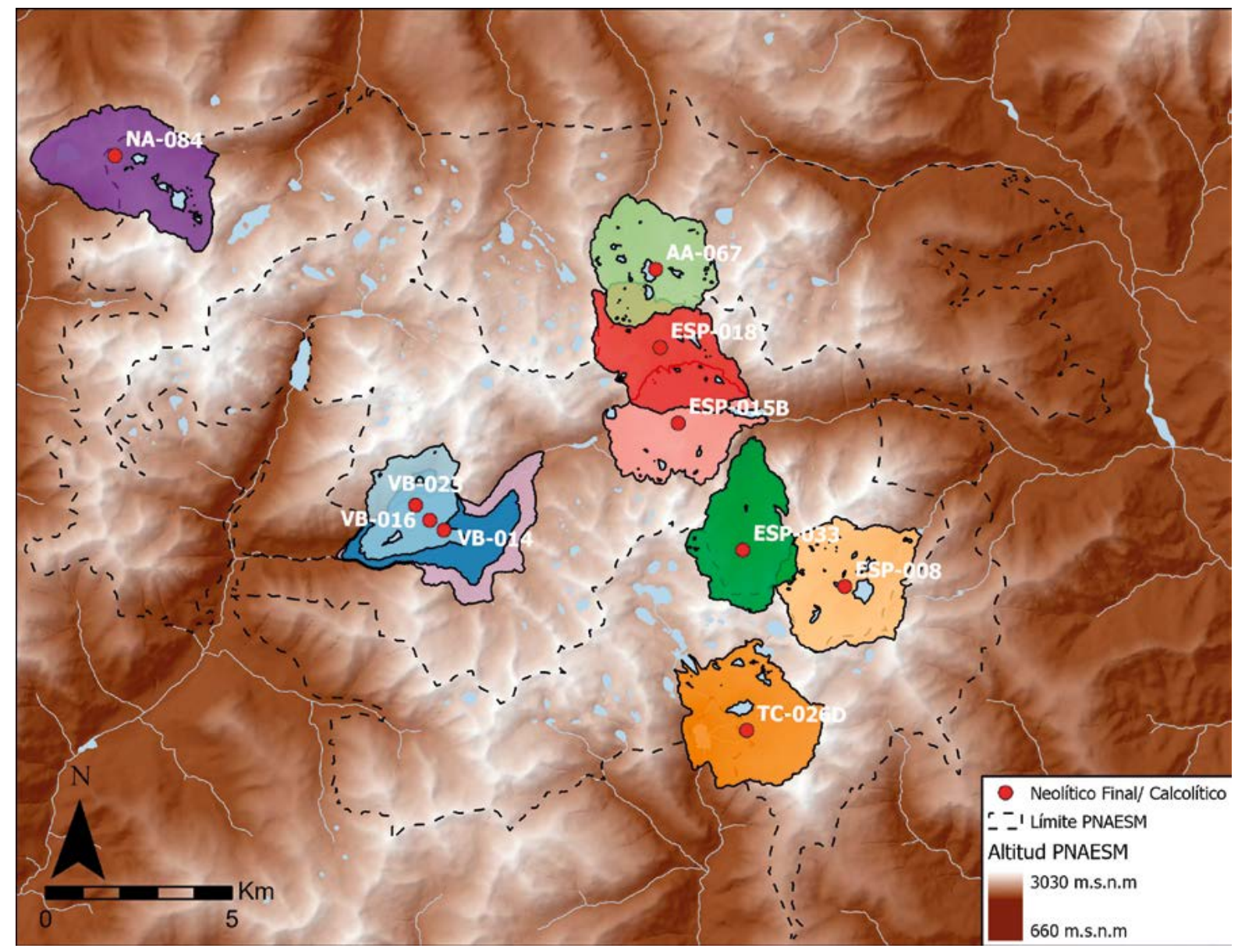

Fig. 5. Parque Nacional d'Aigüestortes i Estany de Sant Maurici (PNAESM). Áreas de influencia de 60 minutos de distancia alrededor de los diferentes yacimientos del Neolítico final/Calcolítico (en color en la versión electrónica).

El área siempre es muy inferior a la que resultaría de proyectar el círculo con $5 \mathrm{~km}$ de radio, típico de los primeros estudios sobre áreas de captación en Arqueología. Las áreas delimitadas por desplazamientos de una hora en torno a los yacimientos prehistóricos tienen poco más de 900 ha de media. Este valor aumenta hasta unas 950 ha en los de época contemporánea. En cada grupo hay, no obstante, una cierta diversidad interna. El coeficiente de variación es de 17,3 en los yacimientos prehistóricos y de 20,6 en los de época contemporánea. Las pruebas estadísticas se han hecho mediante el programa Past 2.17c. El test Shapiro-Wilk indica para ambos grupos distribuciones normales $(\mathrm{w}=0,9691, \mathrm{p}($ normal $)=0,8822 \mathrm{y} \mathrm{w}=$ $0,9079$, $p($ normal $)=0,2672$ respectivamente $)$. La variabilidad en la superficie de terreno accesible entre yacimientos responde, en general, a diferencias en las orografías locales, siendo los terrenos más abruptos los que más dificultan los desplazamientos. En prome- dio la extensión de las áreas de influencia de los yacimientos de época contemporánea es algo superior. Esta diferencia responde principalmente a los valores elevados de un único asentamiento contemporáneo (TC-023), donde la orografía circundante es menos abrupta. De hecho, al excluir este yacimiento, los valores de la prueba Shapiro-Wilk refuerzan esta normalidad $(\mathrm{W}=0,9444, \mathrm{p}($ normal $)=0,6244)$. Apoyan esta consideración el T-test para las medias $(\mathrm{t}=-0,54203$; $\mathrm{p}=0,59444)$ y la prueba de Mann-Whitney para las medianas. Según sus resultados no hay diferencias significativas $(p=0,9697)$ entre las extensiones de las áreas de influencia de los yacimientos del final del Neolítico y de época contemporánea.

A partir de aquí, se han obtenido también las superficies actuales de pastos comprendidas en los recorridos de una hora en torno a los yacimientos (Tab. 2). La superficie media de pastos actuales accesibles desde los yacimientos neolíticos es de 320,3 ha, con una 


\begin{tabular}{|c|c|c|c|c|}
\hline Yacimiento & Cronología & Superficie ha & Pasto ha & $\%$ pasto \\
\hline AA-067 & NEO & 807,81 & 389,10 & 48,17 \\
\hline ESP-008 & NEO & 974,90 & 376,11 & 38,58 \\
\hline ESP-015B & NEO & 891,65 & 278,38 & 31,22 \\
\hline ESP-018 & NEO & 962,94 & 345,08 & 35,84 \\
\hline ESP-033 & NEO & 836,76 & 279,10 & 33,35 \\
\hline NA-084 & NEO & 1119,93 & 608,14 & 54,30 \\
\hline TC-026D & NEO & 1031,57 & 457,93 & 44,39 \\
\hline VB-014 & NEO & 1059,01 & 182,82 & 17,26 \\
\hline VB-016 & $\mathrm{NEO}$ & 763,68 & 143,34 & 18,77 \\
\hline VB-023 & NEO & 600,45 & 143,08 & 23,83 \\
\hline NA-054 & CONT & 1171,02 & 558,17 & 47,66 \\
\hline NA-102 & CONT & 791,13 & 608,14 & 76,87 \\
\hline SO-005 & CONT & 978,09 & 514,44 & 52,60 \\
\hline SO-007A & CONT & 944,70 & 644,17 & 68,19 \\
\hline SO-008B & CONT & 943,48 & 605,09 & 64,13 \\
\hline TC-009 & CONT & 809,30 & 295,61 & 36,53 \\
\hline TC-023 & CONT & 1363,65 & 600,22 & 44,02 \\
\hline VB-004 & CONT & 833,96 & 411,68 & 49,36 \\
\hline VB-017inf & CONT & 949,14 & 303,85 & 32,01 \\
\hline VB-111 & CONT & 694,06 & 251,06 & 36,17 \\
\hline
\end{tabular}

Tab. 2. Superficie total y de pastos en alcanzable desde cada yacimiento documentado en el Parque Nacional d'Aigüestortes i Estany de Sant Maurici en una hora de recorrido. NEO Neolítica; CONT Contemporánea.

desviación típica de 147,3. De promedio supone algo más de un tercio (el 34,6\%) de la superficie accesible alrededor del yacimiento a una hora de recorrido. En los asentamientos de época contemporánea este valor es más elevado: la superficie de pastos a una hora de distancia asciende a 479,2 ha, con una desviación típica de 150,1. En consonancia con este incremento, esta cifra supone casi el 50,8\% de media de la cantidad total de terreno a menos de una hora de cada uno de estos emplazamientos. La diferencia entre ambas series de datos es significativa según los T-test $(\mathrm{t}=-2,3894 ; \mathrm{p}=0,0295)$ y Mann-Withney $(\mathrm{p}=0,049)$.

\subsection{Asentamientos, caminos y movilidad}

Según la cartografía del Departament d'Agricultura (2013), hay tres caminos ganaderos que cruzan los Pirineos de sur a norte en los alrededores del PNAESM. Dos de ellos bordean sus límites actuales por el este y por el oeste. El tercero remonta el valle de Boí y se ramifica a la altura del Estany de Travessani para alcanzar el Valle de Aran cruzando los puertos del Coret de Uelhicrestada y Caldes y descender por los valles de los ríos Rius y Rencules, uniéndose de nuevo en su confluencia antes de llegar a Arties.

La modelización de caminos óptimos se ha efectuado con la finalidad de complementar un hipotético mapa de vías de circulación que amplíe la cartografía actual de vías ganaderas y que ayude a evaluar la disposición de los yacimientos arqueológicos de la zona. El resultado se presenta en la figura 3. Como se puede apreciar, el trazado de los caminos ganaderos actuales en el interior del Parque Nacional coincide en gran medida con el de los caminos teóricos definidos mediante la modelización espacial. La divergencia del camino oriental se debe a la presencia de una pista forestal abierta en el siglo XX y que actualmente se aprovecha también para mover rebaños. Otras discrepancias en algunos puntos de los caminos central y occidental, ya en el Valle de Aran, se explican por la voluntad de evitar determinadas áreas de fondo de valle. Por otra parte, el recorrido de los caminos óptimos coincide también con los senderos que actualmente transcurren por esta área del Pirineo, muy frecuentada por excursionistas. 
La tabla 3 presenta las distancias en minutos de los yacimientos documentados con respecto a las rutas óptimas definidas, así como los caminos pecuarios reconocidos en la cartografía de la Generalitat de Catalunya. Hay una diferencia evidente en la relación de los yacimientos con los caminos óptimos en función de su cronología. De los 10 asentamientos con ocupaciones del Neolítico final/Calcolítico considerados 8 se localizan a menos de 20 minutos de una vía de circulación potencial. Esta situación es inversa en los de época contemporánea: en 8 casos esta distancia supera la media hora de tiempo de desplazamiento. De hecho, 7 de los 10 yacimientos contemporáneos se localizan por encima de la distancia máxima de los asentamientos prehistóricos con relación a los caminos óptimos. Esto se refleja en los respectivos promedios, con una distancia media de poco menos de 12 minutos en los ejemplos del final del Neolítico/Calcolítico y de casi 49 minutos en los contemporáneos. En estos últimos la desviación típica es considerable (32,7 minutos) porque en dos casos el camino óptimo transcurre

\begin{tabular}{|l|c|c|c|}
\hline Yacimiento & Cronología & $\begin{array}{c}\text { Caminos } \\
\text { óptimos }\end{array}$ & $\begin{array}{c}\text { Rutas } \\
\text { cabañeras }\end{array}$ \\
\hline AA-067 & NEO & 31,0 & 138,6 \\
\hline ESP-008 & NEO & 10,2 & 93,0 \\
\hline ESP-015B & NEO & 1,8 & superior a 3 h \\
\hline ESP-018 & NEO & 1,9 & 138,0 \\
\hline ESP-033 & NEO & 2,5 & superior a 3 h \\
\hline NA-084 & NEO & 10,7 & 75,4 \\
\hline TC-026D & NEO & 6,7 & 101,7 \\
\hline VB-014 & NEO & 3,7 & 106,3 \\
\hline VB-016 & NEO & 16,2 & 108,5 \\
\hline VB-023 & NEO & 34,0 & 117,4 \\
\hline NA-054 & CONT & 35,7 & 36,6 \\
\hline NA-102 & CONT & 2,6 & 28,3 \\
\hline SO-005 & CONT & 54,9 & 52,0 \\
\hline SO-007A & CONT & 79,0 & 7,2 \\
\hline SO-008B & CONT & 84,4 & 23,1 \\
\hline TC-009 & CONT & 30,4 & superior a 3 h \\
\hline TC-023 & CONT & 0,1 & 130,4 \\
\hline VB-004 & CONT & 90,9 & 169,6 \\
\hline VB-017inf & CONT & 39,6 & 149,8 \\
\hline VB-111 & CONT & 72,0 & 120,7 \\
\hline
\end{tabular}

Tab. 3. Parque Nacional d'Aigüestortes i Estany de Sant Maurici: distancia en minutos desde los yacimientos al camino óptimo más cercano y a la cabañera más próxima. NEO Neolítica; CONT Contemporánea. muy cerca, o incluso por encima, del asentamiento considerado. En los casos prehistóricos, esta misma desviación no llega a 12 minutos. La diferencia entre ambas es altamente significativa $(\mathrm{t}=-3,3739, \mathrm{p}=$ 0,0044, según el T-test, $\mathrm{p}=0,021$ según el test de Mann-Whitney).

En cambio la situación es notablemente distinta, si se considera la distancia hacia las cabañeras documentadas en la cartografía pecuaria actual. La distancia media de los diferentes yacimientos se incrementa considerablemente. La excepción es SO-007A, localizada bastante cerca de una pista forestal que actualmente cumple también esta función. Por la otra, los yacimientos prehistóricos vuelven a mostrar una pauta claramente propia con relación a los de época contemporánea. Ningún asentamientos del final del Neolítico se emplaza a menos de una hora de distancia de una cabañera, 6 se sitúan a una y dos horas, 2 entre 2 y 3 horas y otros 2 por encima de las $3 \mathrm{~h}$. En cambio, la mitad de los yacimientos de los últimos siglos se localizan a menos de una hora de una vía pecuaria actual y solo uno a más de 3 horas de desplazamiento.

\section{DISCUSIÓN}

La información procesada en este trabajo completa la documentación obtenida en superficie de yacimientos arqueológicos con pequeños sondeos que han permitido fecharlos. Sobre esta base se fechan la práctica totalidad de los asentamientos asignados al final del Neolítico/Calcolítico y una parte de los de época contemporánea. El estudio de algunos materiales y analogías formales complementan la asignación temporal, especialmente para los ejemplos recientes. Todos los vestigios documentados, tanto por su morfología como por su ubicación espacial, se interpretan como asentamientos asociados a la utilización ganadera de esta área de alta montaña. En los contextos prehistóricos esta caracterización se refuerza con los resultados de la excavación del Abric de les Obagues de Ratera de los años 2015 a 2017 (en gran medida inédita, pero con un avance en Gassiot y Clemente 2018), del Abric de l'Estany de la Coveta I (Gassiot 2016) y con la información procedente de contextos similares en otros ámbitos del Pirineo (Dumontier et al. 2016; Gassiot et al. 2017; Orengo et al. 2014; Rendu 2003). La explicación de los asentamientos contemporáneos remite en gran medida a analogías etnográficas, fuentes orales y a la comparación con otros casos pirenaicos de estudio (Garcia Casas 2018; Le Couédic 2010).

Una primera impresión sugiere una cierta identidad entre los registros neolíticos y los contemporáneos salvadas las diferencias arquitectónicas entre los yacimientos de ambos períodos. Se localizan a altitudes

Trab. Prehist., 77, N. ${ }^{\circ}$ 1, enero-junio 2020, pp. 48-66, ISSN: 0082-5638

https://doi.org/10.3989/tp.2020.12246 
relativamente similares, mayoritariamente cerca o ligeramente por encima del actual límite superior del bosque, en lugares cercanos al agua y adyacentes o dentro de actuales zonas de pasto. Siguiendo este hilo, se podría plantear que en el tramo final del Neolítico en esta zona del Pirineo se configuró un sistema de explotación de las zonas altas con cierta analogía con la ganadería de época contemporánea. Si a ello le sumamos la destacada presencia de materiales alóctonos entre las materias primas líticas de los contextos prehistóricos (Mazzucco et al. 2019), la ecuación parece cerrarse. Nos encontraríamos con el surgimiento, a partir del final del IV milenio cal ANE, de un sistema de poblamiento estacional del área del PNAESM, orientado al aprovechamiento de sus ecosistemas alpinos, principalmente como pastos, en el marco de un sistema ganadero con ciertas semejanzas al pastoreo de época histórica. Siguiendo este argumento, cobraría sentido la analogía etnográfica con las informaciones de finales del siglo XIX e inicios del siglo XX para interpretar estos registros prehistóricos.

No obstante, una revisión con mayor profundidad de los registros arqueológicos y paleoambientales matiza de forma relevante esta posibilidad. En otros trabajos ya hemos expuesto las variaciones en la secuencia arqueológica en el PNAESM a lo largo del Holoceno $\mathrm{y}$, en especial, desde el final del Neolítico hasta nuestros días (Gassiot et al. 2014, 2017; Gassiot 2016; Garcia Casas 2018). El vacío de asentamientos durante el II y I milenios cal ANE indica cambios en los patrones que definieron la presencia humana en el área, que excluyen una continuidad lineal hasta la época contemporánea en las pautas del poblamiento. A su vez, los procesos de deforestación y modificación de la cubierta vegetal por causas antrópicas, tampoco son continuos ni progresivos. En aparente paradoja, se intensifican en diversos momentos del II y I milenios cal ANE coincidiendo con la disminución de indicios arqueológicos de asentamientos humanos (Gassiot et al. 2014).

El análisis espacial planteado en este trabajo profundiza en las singularidades de los patrones de dispersión de los yacimientos de dos períodos bien diferenciados: el final del Neolítico o Calcolítico y la época contemporánea. Lejos de representar el inicio y el final de un sistema ganadero de muy larga duración, se documentan importantes diferencias en las variables que definen los emplazamientos de los asentamientos de uno y otro período. Estas divergencias se relacionan con el acceso a pastos y a las vías de circulación. Ambas variables son centrales en las prácticas ganaderas de montaña cuya movilidad depende del agotamiento de las reservas de hierba y los ciclos estacionales

Este trabajo ha tratado de modelizar espacialmente ambas, con todas las cautelas que implica manejar información geográfica y cartografía actuales. Cons- cientemente se ha prescindido de la variabilidad climática holocénica que conllevó oscilaciones de temperatura y pluviosidad mayores de lo supuesto hasta hace pocos años (Catalan et al. 2013). A menudo operó sobre períodos breves de tiempo y de forma relativamente brusca y se desconoce con precisión su efecto en los diferentes ecosistemas del área de estudio. La ausencia de una modelización de detalle de la paleovegetación para cada época y en cada área del PNAESM explica que esta variable tampoco se tuviera en cuenta ni al definir las áreas de influencia alrededor de los yacimientos ni al configurar los caminos óptimos. En ambos casos, no obstante, se han introducido comprobaciones para validar la veracidad de los resultados obtenidos, tanto en las distancias de coste como en las rutas definidas.

Finalmente, se han considerado las áreas de pasto actuales, reflejo de su momento de máxima extensión al ser todavía muy lento el proceso de regeneración forestal vinculado al descenso de la ganadería de la segunda mitad del siglo XX. Los pastos actuales deben reflejar con cierta precisión las áreas de pastoreo en torno a los asentamientos de época reciente. Esta asociación es más problemática para los de cronología prehistórica. En todo caso, pueden reflejar un umbral cercano al máximo de extensión de los pastos a su alrededor que, en caso de una mayor forestación, sería sensiblemente menor. De haberse producido, las inferencias que siguen cobrarían todavía mayor fuerza.

De los resultados obtenidos se desprenden dos argumentos centrales. El primero es la mayor presencia de pastos alrededor de los asentamientos de época contemporánea en comparación con los del final del Neolítico/Calcolítico. La extensión de las áreas de una hora de desplazamiento alrededor de los asentamientos de ambos períodos tiende a ser similar, mientras la extensión de las superficies de pastos es casi un $50 \%$ superior de media en los yacimientos de época histórica. Este mayor acceso directo a la hierba se correlaciona con la mayor lejanía de estos mismos asentamientos a las vías óptimas de circulación que, como se ha detallado más arriba, en gran medida se corresponden con los senderos actuales de excursionistas. Es decir, el patrón de asentamiento del siglo XVIII y especialmente del siglo XIX e inicios del siglo XX parece priorizar la obtención de hierba para el ganado $y$, con ello, sacrifica la accesibilidad a los lugares donde se definen los refugios de personas y animales. La imagen descrita coincide en gran medida con la información etnográfica.

En cambio, al final de la época neolítica el patrón cambia significativamente. Desde cada asentamiento se accede de forma rápida y directa a una superficie notablemente menor de pastos. Es decir, o menos ganado podía pacer en un radio inmediato al lugar don- 
de pernoctaban las personas que lo controlaban o lo podían hacer durante menos tiempo. Por el contrario, la accesibilidad de los lugares de refugio y pasto era más sencilla y se vinculaba de forma más directa a lo que pudo haber sido la red de circulación viaria en este sector del Pirineo. Ello es coherente con la imagen general de movilidad que proporciona la arqueología para las ocupaciones de este período: uso reiterado de los espacios de hábitat con ocupaciones cortas, considerable presencia de materiales exógenos, a veces procedentes de distancias superiores a los $50 \mathrm{~km}$, etc. (Gassiot 2016; Mazzucco et al. 2019).

Llegados a este punto, salta a la vista otro aspecto de interés. Sin la menor duda los registros arqueológicos de superficie del PNAESM son todavía parciales. Sin embargo es notoria la disposición de 6 de los yacimientos prehistóricos considerados sobre una de las vías de tránsito sur-norte en la mitad oriental del parque. Por otra parte, las distancias entre ellos son relativamente constantes (Tab. 3). Todos estos yacimientos comparten fases de ocupación del final del IV e inicios del III milenio cal ANE. No distan mucho unos de otros y tienen, al menos, un asentamiento de la misma época en un rango próximo y, en general, algo inferior a las 2 horas de desplazamiento. De hecho, en cinco casos el área de influencia de una hora se solapa en parte, al menos, con el de otro yacimiento de la misma época. Se desprende así una cierta regularidad en la disposición de los abrigos con ocupaciones del final del Neolítico/ Calcolítico en la mitad oriental del área de estudio, tanto en la distancia entre ellos como en su asociación con rutas de paso en sentido sur-norte.

Este último aspecto abre la puerta a otra cuestión. Desde una perspectiva diacrónica, se aprecia un claro incremento de la cifra de asentamientos conocidos al final del Neolítico tanto en el PNAESM como en otros sectores del Pirineo (Gassiot et al. 2017). Este fenómeno se traduce en un mayor número de ocupaciones registradas y datadas mediante $\mathrm{C} 14$. Paleoecológicamente coincide con el inicio de un impacto antrópico en la vegetación que, no obstante, es todavía leve en comparación con las magnitudes observadas en los milenios posteriores. Una lectura posible de la confluencia de ambas situaciones lleva a argumentar un incremento de la población en la zona, con una intensificación del aprovechamiento ganadero del piso alpino y la parte superior del subalpino. El incremento del número de asentamientos a partir del $3300 \mathrm{cal}$ ANE podría reflejar una ocupación simultánea de las pequeñas cuencas en las cabeceras de los diferentes valles del PNAESM. Otra alternativa puede radicar en una ocupación menos intensa del espacio por parte de grupos bastante móviles, que desplazarían sus refugios en diversas ocasiones a lo largo de la época cálida y sin nieve. En este caso, el volumen de pasto que circunda cada asentamiento sería sensiblemente menor. Esta opción parece corresponder mejor con indicios todavía tenues de antropización de la paleovegetación y con el escaso volumen de materiales muebles presentes en las ocupaciones de este período.

\section{CONCLUSIONES}

El estudio de las sociedades humanas del pasado a través de la disposición espacial de sus restos materiales es una tendencia en Arqueología que, desde hace unas décadas, ha aportado gran cantidad de datos y modelos interpretativos para diversos periodos prehistóricos e históricos, así como regiones (Conolly y Lake 2009). Más allá del estudio arqueológico centrado en el yacimiento como unidad básica y final de la investigación, el análisis de las relaciones espaciales de los restos materiales tanto entre sí como con su entorno natural y social permite comprender mejor la vertiente geográfica de la actividad humana en el pasado. De esta manera se pueden elaborar explicaciones sobre las circunstancias que envuelven el asentamiento de los grupos humanos en un determinado lugar y comprender la variación de estos patrones a lo largo del tiempo. Además, permite entender las relaciones de los grupos humanos con su entorno más inmediato y su estructuración del espacio en función de sus prácticas sociales.

El presente trabajo representa la aplicación de estas líneas de investigación a una zona de alta montaña donde en los últimos años se han documentado numerosos restos arqueológicos en un espacio continuo. La localización de los asentamientos prehistóricos próximos entre sí y a la vez relativamente cerca de una posible ruta de comunicación entre valles muestra una estructuración del espacio muy diferente a la de los últimos siglos, cuando la cantidad de pasto alrededor del sitio parece cobrar mucho más peso. Los territorios de montaña, en época moderna y contemporánea, estaban, y están, parcelados y delimitan de modo muy preciso los derechos de pasto. Por el contrario, la organización de la montaña en el Neolítico final parece muy distinta. Estos resultados complementan otras investigaciones arqueológicas en el PNAESM que desmienten una supuesta continuidad entre la ganadería prehistórica y la trashumancia tradicional documentada por los etnógrafos a principios del siglo XX (García Casas 2018; Gassiot et al. 2014; Gassiot 2016; Mazzucco et al. 2019). Los resultados expuestos son de carácter parcial y han de considerarse junto con estudios arqueológicos y paleoecológicos más amplios. Sin embargo muestran cómo el análisis espacial proporciona herramientas para modelizar los factores que definieron las formas de vida en los Pirineos en la Prehistoria.

Trab. Prehist., 77, N. ${ }^{\circ}$ 1, enero-junio 2020, pp. 48-66, ISSN: 0082-5638

https://doi.org/10.3989/tp.2020.12246 


\section{AGRADECIMIENTOS}

En el trabajo de campo se ha contado con la participación indispensable de los miembros de GAAM (Grup d'Arqueologia de l'Alta Muntanya), así como de numerosos estudiantes del Dpto. de Prehistoria de la Universidad Autónoma de Barcelona. La investigación expuesta no habría sido posible sin la colaboración y el apoyo tanto de la dirección como de los trabajadores del PNAESM.

\section{ANEXO: MATERIAL COMPLEMENTARIO}

En la versión electrónica de este artículo, disponible en libre acceso en la página web de la revista se incluye en el PDF un anexo que detalla la explicación del algoritmo inédito utilizado para el cálculo del tiempo de desplazamiento. Explica los cálculos de base de la fórmula y la comparación con los resultados derivados de otros algoritmos utilizados en arqueología.

\section{CARTOGRAFÍA}

Centre de Recerca Ecologica i Aplicacions Forestals 2013. Mapa de Cobertes del Sol Versió 4 (MSCS-4). http://www.creaf.uab.es/mcsc/ (consulta 22-02-2017)

Departament de Medi Ambient y Agència Catalana de l'Aigua 2015. Xarxa hidrográfica. http://aca-web.gencat.cat/aca (descarga 21-022017).

Departament d'Agricultura, Ramaderia, Pesca i Alimentació 2013. Camins ramaders classificats. http://agricultura.gencat.cat/ca/detalls/ Article/Camins-ramaders (consulta 21-02-2017).

Institut Cartogràfic i Geològic de Catalunya 2013. Model d'Elevacions del Terreny de Catalunya 5 x 5 metres https://www.icgc.cat/ Descarregues/Elevacions/Model-d-elevacions-del-terreny-de-5x5-m (descarga 21-02-2017).

\section{BIBLIOGRAFÍA}

Bevan, A. 2011: "Computational models for understanding movement and territory”. En V. Mayoral y S. Celestino (eds.): Tecnologías de información geográfica y análisis arqueológico del territorio: Actas del $V$ Simposio Internacional de Arqueología de Mérida. Anejos de Archivo Español de Arqueología LIX, CSIC. Mérida: 383-394.

Calastrenc, C.; Le Couedic, M. y Rendu, C. 2006: "Archéologie pastorale en vallée d'Ossau. Problématiques, méthodes et premiers résultats". Archéologie des Pyrénées Occidentales et des Landes 25: $12-$ 30.

Carrer, F. 2013: “An ethnoarchaeological inductive model for predicting archaeological site location: A case-study of pastoral settlement patterns in the Val di Fiemme and Val di Sole (Trentino, Italian Alps)". Journal of Anthropological Archaeology 32 (1): 54-62. https://doi.org/10.1016/j.jaa.2012.10.001

Catalan, J.; Pèlachs, A.; Gassiot, E.; Antolín, F.; Ballesteros, A.; Batalla, M.; ... y Soriano, J. M. 2013: "Interacción entre clima y ocupación humana en la configuración del paisaje vegetal del Parque Nacional de Aigüestortes i Estany de Sant Maurici a lo largo de los últimos 15.000 años". En L. Ramírez y B. Asensio (eds.): Proyectos de investigación en Parques Nacionales: 2009-2012 - Naturaleza y Par- ques Nacionales. Organismo Autónomo de Parques Nacionales. Madrid: 71-92.

Clemente-Conte, I.; Gassiot Ballbè, E.; Rey Lanaspa, J.; Antolín Tutusaus, F.; Obea Gómez, L.; Viñerta Crespo, A. y Saña Segui, M. 2016: "Cueva de Coro Trasito (Tella-Sin, Huesca): un asentamiento pastoril en el Pirineo Central con ocupaciones del Neolítico Antiguo y del Bronce Medio". En J. I. Lorenzo Izalde y J. M. Rodanés Vicente (eds.): I Congreso CAPA, Arqueología Patrimonio Aragonés (Zaragoza 2015): 74-83. Zaragoza.

Conolly, J. y Lake, M. 2009: Sistemas de información geográfica aplicados a la arqueología. Bellaterra arqueología. Barcelona.

Díaz Bonilla, S.; Clemente-Conte, I.; Gassiot, E.; García, D.; Rodríguez, D.; Obea, L.... y Rey, J. 2016: “Arqueología y patrimonio en la alta montaña. Resultado de las prospecciones en el valle de Góriz (Fanlo, Huesca)". En J. I. Lorenzo Izalde y J. M. Rodanés Vicente (eds.): I Congreso CAPA, Arqueología Patrimonio Aragonés (Zaragoza 2015): 643-650. Zaragoza.

Dumontier, P.; Courtrand, P.; Armand, D.; Convertini, F. y Ferrier, C. 2016: "Entre montagne et piémont, témoignes agropastoraux du Néolithique à l'âge du Fer". En C. Rendu, C. Calastrenc, M. Le Couédic y A. Berdoy (ed.): Estives d'Osseau. 7000 ans de pastoralisme dans les Pyrénées. Le Pas d'Oiseau. Tarbes: 175-203

Galop, D.; Carozza, L.; Marembert, F. y Bal, M. C. 2007: "Activités agropastorales et climat durant l'Âge du Bronze dans les Pyrénées: l'état de la question à la lumière des données environnementales et archéologiques”. En H. Richard, M. Magny y C. Mordant (eds.): Environnements et cultures a l'âge du Bronze en Europe occidentale. CTHS. Paris: 107-119.

Garcia Casas, D. 2013: “Aproximación al poblamiento de las zonas de alta montaña pirenaicas desde la arqueología y la etnografía”. Saguntum. Papeles del Laboratorio de Arqueología de Valencia 45: 221239. https://doi.org/10.7203/sagvntvm.45.2006

Garcia Casas, D. 2018: Arqueologia d'un territori d'alta muntanya del Pirineu Central. Persones, ramats i prats al llarg de la història al Parc Nacional d'Aigüestortes i Estany de Sant Maurici. Tesis doctoral. Universidad Autónoma de Barcelona. Bellaterra. https://hdl.handle.net/10803/666995

Gassiot, E. (ed.) 2016: Arqueología del pastoralismo en el Parque Nacional d'Aigüestortes i Estany de Sant Maurici Montañas humanizadas. Organismo Autónomo Parques Nacionales. Madrid.

Gassiot, E. y Clemente, I. 2018: "Unes muntanyes amb gent, ramats i camps". En G. Remolins y J. F. Gibaja (eds.): Les Valls d'Andorra durant el Neolitic: un encreuament de camins al centre dels Pirineus. Monografies del Museu d'Arqueologia de Catalunya 2. Barcelona: 63-75.

Gassiot, E.; Clemente, I.; Mazzucco, N.; Garcia Casas, D.; Obea, L. y Rodriguez-Antón, D. 2016: "Surface surveying in high mountain areas, is it possible? Some methodological considerations". Quaternary International 402: 35-45. https://doi.org/10.1016/j.quaint.2015.09.103

Gassiot, E.; Mazzucco, N.; Clemente-Conte, I.; Rodríguez-Antón, D.; Obea, L.; Quesada, M. y Díaz, S. 2017: "The beginning of high mountain occupations in the Pyrenees. Human settlements and mobility from 18,000 cal BC to 2000 cal BC". En J. Catalan, J. Ninot, M. Aniz (eds.): High mountain conservation in a changing world. Springer. Cham: 75-105.

Gassiot, E.; Mazzucco, N.; Obea, L.; Tarifa, N.; Antolín, F.; Clop, X... y Saña, M. 2015: “La Cova del Sardo de Boí i l'explotació de l'alta muntanya als Pirineus occidentals en època neolítica”. Tribuna d'arqueologia 2012-2013: 199-218.

Gassiot, E.; Rodríguez-Antón, D.; Pèlachs, A.; Pérez Obiol, R.; Julià, R.; Bal, M. C. y Mazzucco, N. 2014: "La alta montaña durante la Prehistoria: 10 años de investigación en el Pirineo catalán occidental". Trabajos de Prehistoria 71 (2): 262-282. https://doi.org/10.3989/tp.2014.12134

Gietl, R.; Doneus, M. y Fera, M. 2008: "Cost Distance Analysis in an Alpine environment: Comparison of different Cost Surface Modules". En A. Posluschny, K. Lamberse e I. Herzog (eds.): Layers of perception. Proceedings of the $35^{\text {th }}$ International Conference on Computer Applications and Quantitative Methods in Archaeology (CAA), 2007 Berlin. Habelt Verlag. Bonn: 336-341. 
Gómez, D. 2008: “Aspectos ecológicos de los pastos". En F. Fillat, R. García González, Y. Gómez y R. Reine (eds.): Pastos del Pirineo. CSIC, Diputación de Huesca. Madrid: 61-74.

González Álvarez, D. 2016: Poblamiento y antropización de la montaña occidental cantábrica durante la Prehistoria reciente: Una aproximación desde la Arqueología del Paisaje. Tesis doctoral. Universidad Complutense de Madrid. Madrid. https://eprints.ucm.es/39363/1/T37836.pdf (consulta 22-02-2019)

Herzog, I. 2013: "The potential and limits of optimal path analysis". En A. Bevan y M. Lake (eds.): Computational Approaches to Archaeological Spaces. Left Coast Press. Walnut Creek: 179-211.

Herzog, I. 2014: "A review of case studies in archaeological least-cost analysis". Archeologia e Calcolatori 25: 223-239.

Hudson, E. J. 2012: "Walking and watching: New approaches to reconstructing cultural landscapes through space syntax analysis". En D. A. White y S. L. Surface-Evans (eds.): Least Cost Analysis of social landscapes. Archaeological case studies. The University of Utah Press. Salt Lake City: 97-108.

Kantner J. 2012: "Realism, reality, and routes". En D. A. White y S. L. Surface-Evans (eds.): Least Cost Analysis of social landscapes. Archaeological case studies. The University of Utah Press. Salt Lake City: 225-238.

Laborda, R.; Villalba-Mouco, V.; Lanau, P.; Gisbert, M.; Sebastián, M.; Domingo, R. y Montes, L. 2017: “El Puerto Bajo de Góriz (Parque Nacional de Ordesa y Monte Perdido). Ocupación y explotación de un paisaje de alta montaña desde la Prehistoria hasta el siglo XX". Bolskan 26: 9-30.

Landais, E. y Balent, G. 1995: "Introduction à l'étude des systèmes d'élevage extensif'. En E. Landais y G. Balent (eds.): Pratiques d'élevage extensif. Identifier, modéliser, évaluer. INRA. Versailles: 13-35.

Langmuir, E. 1995: Mountaincraft and leadership. MLTUK. London and Edinburgh.

Le Couédic, M. 2010. Les pratiques pastorales d'altitude dans une perspective ethnoarchéologique. Cabanes, troupeaux et territoires pastoraux pyrénéens dans la longue durée. Université François-Ravelais de Tours. http://www.sudoc.fr/153137428

Le Couédic, M. 2012: "Modéliser les pratiques pastorales d'altitude dans la longue durée". Cybergéo: European Journal of Geography, Systèmes, Modélisation, Géostatistique, doc. 590. https://doi.org/10.4000/cybergeo.25123

Marín Arroyo, A. B. 2008: "Patrones de movilidad y control del territorio en el Cantábrico oriental durante el Tardiglaciar". Trabajos de Prehistoria 65 (1): 29-45.

Marín Arroyo, A. B. 2009: "The use of Optimal Foraging Theory to estimate Late Glacial site catchment areas from a central place: The case of Eastern Cantabria, Spain". Journal of Anthropological Archaeology 28 (1): 27-36. https://doi.org/10.1016/j.jaa.2008.11.001

Mazzucco, N.; Clemente, I. y Gassiot, E. 2019: "Lost in the mountains? The Cova del Sardo and the Neolithisation of the Southern Central
Pyrenees (fifth-third mill. cal BC)". Archaeological and Anthropological Sciences 11 (4): 1461-1475. https://doi.org/10.1007/s12520-018-0603-0

Orengo, H. A.; Palet, J. M.; Ejarque, A.; Miras, Y. y Riera, S. 2014: "Shifting occupation dynamics in the Madriu-Perafita-Claror valleys". Quaternary International 353 (5): 140-152. https://doi.org/10.1016/j.quaint.2014.01.035

Pèlachs, A.; Pérez-Obiol, R.; Soriano, J. M.; Cunill, R.; Bal, M. C. y García-Codron, J. C. 2017: "The Role of Environmental Geohistory in High-Mountain Landscape Conservation". En J. Catalan, J. M. Ninot y M. Aniz (eds.): High Mountain Conservation in a Changing World. Springer Open, Cham: 107-129. https://link.springer.com/chapter/10.1007/978-3-319-55982-7 5

Reimer, P. J.; Bard, E.; Bayliss, A.; Beck, J. W.; Blackwell, P. G.; Ramsey C. B. and Van der Plicht, J. 2013: "IntCal13 and Marine13 radiocarbon age 22 calibration curves 0-50,000 years cal BP". Radiocarbon 55 (4): 1869-1887. https://doi.org/10.2458/azu js rc.55.16947

Rendu, C. 2003: La montagne d'Enveig, une estive pyrénéenne dans la longue durée. Trabucaire. Canet-sur-mer.

Rendu, C.; Calastrenc, C.; Le Couédic, M. y Berdoy, A. (eds.) 2016: Estives d'Osseau. 7000 ans de pastoralisme dans les Pyrénées. Le Pas d'Oiseau. Tarbes

Rojo, M.; Peña-Chocarro, L.; Royo, I.; Tejedor, C.; Martínez de Lagrán, I.; Arcusa, H... y Alt, K. 2013: "Pastores trashumantes del Neolítico Antiguo en un entorno de alta montaña: secuencia crono-cultural de la Cova de Els Trocs, San Feliú de Veri (Huesca)". Boletín del Seminario de Estudios de Arte y Arqueología, Arqueología 13: 9-54.

Sánchez, A.; Domínguez-Ballesteros, E.; García-Rojas, M.; Prieto, A.; Calvo, A. y Ordoño, J. 2016: "Patrones de aprovisionamiento de sílex de las comunidades superopaleolíticas del Pirineo occidental: el 'coste' como medida de análisis a partir de los SIG'. Munibe Antropologia-Arkeologia 67: 235-252. https://doi.org/10.21630/maa.2016.67.mis02

Tobler, W. 1993: Three presentations of geographical analysis and modelling. Thechnical Report 93-1, University of California. Santa Barbara.

Vilarrassa, S. 1981: La vida dels pastors. Maideu. Ripoll.

Violant, R. 1949: El Pirineo Español: vida, usos, costumbres creencias $y$ tradiciones de una cultura milenaria que desaparece. Plus Ultra. Madrid.

Violant, R. 2001: La vida pastoral al Pallars. Garsineu. Tremp.

Vita-Finzi, C. y Higgs, E. S. 1970: "Prehistoric economy in the Mount Carmel area of Palestine: site catchment analysis". Proceedings of the prehistoric Society 36: 1-37.

Wheatley, D. y Gillings, M. 2002: Spatial technology and Archaeology: The archaeological applications of GIS. CRC. London.

White, D. A. y Surface-Evans, S. L. (eds.). 2012: Least Cost Analysis of social landscapes. Archaeological case studies. The University of Utah Press. Salt Lake City. 